\title{
DOES ANTHROPOMETRY INFLUENCE TECHNICAL FACTORS IN COMPETITIVE MIXED MARTIAL ARTS?
}

original paper

( ) University School of Physical Education in Wroclaw

DOI: https://doi.org/10.5114/hm.2018.74059

\section{CHRISTOPHER KIRK}

University of Derby, Derby, United Kingdom

\begin{abstract}
Purpose. Previous research has found grappling and strikes to the head to be the determining factors for success in MMA, whilst anthropometry in the form of stature and wingspan has been found to have a negligible effect. The current study was designed to determine if a relationship between technique use and anthropometry exists in MMA.

Methods. The in-competition technique data of 461 elite, professional MMA bouts were compared using Bayes factor t-tests $\left(\mathrm{BF}_{10}\right)$ to determine which techniques display the highest likelihood of distinguishing between winners and losers. The differences in technique numbers between winners and losers was also compared to the anthropometric differences between winners and losers using either Bayesian Pearson's r or Bayesian Kendall's Tau. Simple linear regression $(p<0.05)$ was used to calculate predictive ability of anthropometrics on chosen fighting techniques.
\end{abstract}

Results. Heavyweight competitors were distinguished by striking only $\left(\mathrm{BF}_{10}=399-10\right)$. Light heavyweight, middleweight, featherweight, bantamweight, women's bantamweight and women's straw weight competitors were distinguished predominately by striking $\left(\mathrm{BF}_{10}=791661-7\right)$ and moderately by grappling $\left(\mathrm{BF}_{10}=75-7\right)$. Welterweight, lightweight and flyweight competitors were distinguished by striking techniques $\left(\mathrm{BF}_{10}=3.533 \mathrm{e}+6-221\right)$ to achieve dominant grappling positions $\left(\mathrm{BF}_{10}=17100-50\right)$. In turn, it was found that stature and/or wingspan are correlated to and can predict key technique variables at heavyweight, welterweight, lightweight and featherweight.

Conclusions. The results provide evidence of which techniques are influenced by the anthropometric differences between competitors, allowing coaches and competitors to make more informed tactical decisions in competition preparation.

Key words: MMA, anthropometry, combat sports, Bayes factor, key performance variables

\section{Introduction}

Mixed martial arts (MMA) is a relatively young combat sport in which the competitors must physically engage their opponent using kicks, punches, elbows and knees along with grappling manoeuvres to overcome their opponent [1, 2]. A competitor is deemed to have won the bout if the referee is given reason to believe their opponent is no longer in a position to defend themselves, the opponent 'submits' to chokes or joint locks, all the scheduled rounds of the bout have been completed and the appointed judges declare the bout winner, or if an appointed doctor determines that either participant is no longer fit to continue safely [3]. MMA participants must be capable of successfully uti- lising a varied skill set including movements incorporated from a variety of combat sports such as boxing, muay Thai, wrestling, judo and Brazilian jiu jitsu $[2,4]$.

Due to a relative lack of published research regarding the physiological or performance parameters of MMA, little is known about the tactical requirements of the participants to maximise the chances of a successful performance [5] although recent studies are beginning to fill this gap in the knowledge [6-10]. Time motion analyses have demonstrated that MMA competitors display a work-to-rest ratio of 1:4 in competition $[6,11]$ and 1:1.01 in training based sparring bouts [12], indicating that in competition participants are likely to spend four times as long in a resting state than in a working state, whereas in sparring the time differ-

Correspondence address: Christopher Kirk, University of Derby, Kedleston Rd, DE22 1GB Derby (Derbyshire), United Kingdom, e-mail: C.Kirk@Altius-Sports.co.uk

Received: September 18, 2017

Accepted for publication: January 15, 2018

Citation: Kirk C. Does anthropometry influence technical factors in competitive mixed martial arts?. Hum Mov. 2018;19(2): 46-59; doi: https://doi.org/10.5114/hm.2018.74059. 
ences between these states is negligible. Amongst the current research which details the occurrence and effectiveness of specific techniques within competition, James et al. [9] provide a decision tree demonstrating that effective grappling and technique accuracy are the determining factors in winning and losing. This standpoint is supported by Kirk et al. [12] who found that successful takedowns were the distinguishing factor between winners and losers in MMA sparring bouts. Similarly, strikes to the head from distance, alongside successfully taking down an opponent and moving to a more dominant position have been found to play a key role in determining the winner of a bout [13]. Whilst James et al. [9] published effect sizes of the mean differences in techniques used between bout winners and losers, there are no studies that elucidate the relative likelihoods of techniques being used by winners and losers, such as those provided by Bayesian statistical analysis as recommended by contemporary statistical authors [14-16]. Knowledge of such likelihoods could provide valuable information for coaches and competitors to further inform training, preparation and tactical approaches to competition. While the referenced studies provide evidence for which techniques and combinations of techniques increase a participant's likelihood of winning, they do not consider the effects of anthropometric differences between participants.

The anthropometry of combat sports participants in relation to stature and arm span (henceforth discussed using the term wingspan, in keeping with the extant MMA related literature) is relatively limited, with studies mainly focussing on lean mass distribution or girth measurements [17-20]. Recent research into the anthropometrical makeup of MMA competitors found that differences in stature, wingspan or stature-to-wingspan ratio (S:W) have negligible ef- fects on who wins or loses an MMA bout, but can influence how the bout is won or lost, with taller competitors having a higher likelihood of losing due to strikes [21]. It has also been demonstrated that a competitor's anthropometry has negligible effect on their divisional ranking or attainment of a world title bout [22], despite a positive effect of anthropometry being found in other sports in which physical engagement is a primary objective [23-26]. Unlike in rugby league or Australian rules football, body mass is generally normalised in MMA using separate weight classes or mass divisions, the same cannot be said for stature or wingspan. In relation to these two variables, whilst anthropometry does not seem to have a large effect on overall success, it has not yet been determined whether it has any effect on the types of techniques used in competition, or the relative importance of these techniques in winning or losing.

This study investigated the following two hypotheses. The first hypothesis was that each division would contain key technique variables in which bout winners would have a higher likelihood of attaining greater numbers than bout losers as recorded in competition. The second hypothesis was that participant anthropometric differences would be positively correlated to, and could be used to predict, the differences between bouts winners and losers in these key technique variables.

\section{Material and methods}

The in bout data of 461 professional MMA bouts were analysed, having been collected and collated by Fightmetric, the official statistical partner of the Ultimate Fighting Championship (UFC) during the period from the $1^{\text {st }}$ of January 2015 to the $31^{\text {st }}$ of December 2015 inclusive. The descriptive statistics of each com-

Table 1. Descriptive statistics of competitive divisions included in this study

\begin{tabular}{|c|c|c|c|c|c|c|}
\hline Competitive Division & $\begin{array}{l}\text { Mass Limit } \\
(\mathrm{kg})\end{array}$ & $\begin{array}{l}\mathrm{N} \text { of Bouts } \\
\text { Included }\end{array}$ & $\begin{array}{c}\text { Bout winner's } \\
\text { stature }(\mathrm{cm})\end{array}$ & $\begin{array}{l}\text { Bout winner's } \\
\text { wingspan }(\mathrm{cm})\end{array}$ & $\begin{array}{l}\text { Bout loser's } \\
\text { stature }(\mathrm{cm})\end{array}$ & $\begin{array}{c}\text { Bout loser's } \\
\text { wingspan }(\mathrm{cm})\end{array}$ \\
\hline Heavyweight (HW) & 120.5 & 28 & $190.4 \pm 5.8$ & $197.1 \pm 7$ & $188.8 \pm 6.9$ & $195.2 \pm 7.9$ \\
\hline Light Heavyweight (LHW) & 93.1 & 25 & $187.4 \pm 3.9$ & $194.1 \pm 7.1$ & $188.2 \pm 4.3$ & $193.9 \pm 7.2$ \\
\hline Middleweight (MW) & 84 & 51 & $185.2 \pm 3.5$ & $191.5 \pm 6.3$ & $185.3 \pm 3.8$ & $190.0 \pm 6.2$ \\
\hline Welterweight (WW) & 77.2 & 87 & $182.3 \pm 4.5$ & $187.2 \pm 6.1$ & $181.9 \pm 4.5$ & $186.9 \pm 5.5$ \\
\hline Lightweight (LW) & 70.5 & 99 & $178.1 \pm 4.7$ & $182.8 \pm 6.2$ & $177.1 \pm 4.2$ & $181.8 \pm 5.4$ \\
\hline Featherweight (FW) & 65.9 & 53 & $175.2 \pm 4.2$ & $179.1 \pm 6.6$ & $175.4 \pm 4.5$ & $178.3 \pm 6.2$ \\
\hline Bantamweight (BW) & 61.3 & 36 & $169.8 \pm 3.4$ & $174.2 \pm 6$ & $170.4 \pm 4.8$ & $173.9 \pm 6$ \\
\hline Flyweight (FlW) & 56.8 & 36 & $165.8 \pm 5.4$ & $168.1 \pm 5.1$ & $166.7 \pm 4.3$ & $168.9 \pm 5.5$ \\
\hline Women's Bantamweight (WBW) & 61.3 & 20 & $169.1 \pm 4$ & $171.5 \pm 5.9$ & $167.7 \pm 4.9$ & $169.1 \pm 6.8$ \\
\hline Women’s Strawweight (WSW) & 52.3 & 24 & $163.2 \pm 4.6$ & $163.5 \pm 5.3$ & $163.8 \pm 4.8$ & $166.3 \pm 5.3$ \\
\hline
\end{tabular}




\section{HUMAN MOVEMENT}

C. Kirk, Does anthropometry influence technical factors in competitive mixed martial arts?

petitive division included in the study can be viewed in Table 1.14 variables were selected for inclusion in the current study, due to these variables being deemed to be a fair representation of the key movements and techniques in MMA as well as being amongst the variables used in previous research, thus allowing effective comparison between findings to occur [9]. The collection protocol used for these data has been described by Kuhn and Crigger [27]. Each technique variable used in this study was found to have high reliability according to Cronbach's $\alpha(0.899-0.923)$. All bouts ending in a draw or a no-contest were ex- cluded from the sample. The chosen technique variables, their abbreviations and definitions are included in Table 2.

The following anthropometric data were recorded for the winner and loser of each bout, using the 'tale of the tape' measurements reported by event promoters: mass $(\mathrm{kg})$; stature $(\mathrm{cm})$; wingspan length $(\mathrm{cm})$. Each participant's stature-to-wingspan ratio (S:W) was calculated using the following formula:

$$
\mathrm{S}: \mathrm{W}=\text { wingspan } / \text { stature. }
$$

Table 2. Technique variables used with abbreviations and definitions

\begin{tabular}{|c|c|c|}
\hline Variable & Abbreviation & Definition \\
\hline Standing & $\mathrm{N} / \mathrm{A}$ & $\begin{array}{l}\text { Where only the participant's soles of the feet are in contact } \\
\text { with the ground. }\end{array}$ \\
\hline Grounded & $\mathrm{N} / \mathrm{A}$ & $\begin{array}{l}\text { Where any other part of the participant's body apart from the } \\
\text { soles of the feet are in contact with the ground }\end{array}$ \\
\hline Clinch & $\mathrm{N} / \mathrm{A}$ & $\begin{array}{l}\text { A standing grappling position where each participant is } \\
\text { attempting to physically control their opponent. }\end{array}$ \\
\hline Power strikes & $\mathrm{N} / \mathrm{A}$ & $\begin{array}{l}\text { Any strike attempted with the intent to cause damage, } \\
\text { as opposed to managing distance or setting up further strikes }\end{array}$ \\
\hline Total strikes landed & StrLanded & $\begin{array}{l}\text { All strikes which successfully make contact with the opponent } \\
\text { regardless of standing, clinch or grounded positions }\end{array}$ \\
\hline Total strikes attempted & StrAtt & $\begin{array}{l}\text { All strikes attempted, regardless of success and regardless } \\
\text { of standing, clinch or grounded positions }\end{array}$ \\
\hline Significant strikes landed & SigStrLanded & $\begin{array}{l}\text { All power strikes which successfully make contact with opponent } \\
\text { regardless of standing, clinch or grounded positions }\end{array}$ \\
\hline Significant strikes attempted & SigStrAtt & $\begin{array}{l}\text { All power strikes attempted, regardless of their success } \\
\text { and regardless of standing, clinch or grounded positions }\end{array}$ \\
\hline Significant distance strikes landed & SigDisStrLan & A power strike landed in a standing, non-clinched position only \\
\hline $\begin{array}{l}\text { Significant distance strikes } \\
\text { attempted }\end{array}$ & SigDisStrAtt & $\begin{array}{l}\text { A power strike attempted in a standing, non-clinched position } \\
\text { only, regardless of success. }\end{array}$ \\
\hline Total knockdowns & KDowns & $\begin{array}{l}\text { Any strike resulting in the opponent becoming grounded } \\
\text { regardless of standing or clinch positions }\end{array}$ \\
\hline Total distance knockdowns & DistKDowns & $\begin{array}{l}\text { Any strike resulting in the opponent becoming grounded from } \\
\text { a standing, non-clinched position only }\end{array}$ \\
\hline Significant clinch strikes landed & SigClinStrLan & A power strike landed in a standing, clinched position only \\
\hline Significant ground strikes landed & SigGrounStrLan & A power strike landed in a grounded position only \\
\hline Successful takedowns & TDSucc & A successful grappling attempt to ground the opponent \\
\hline Attempted takedowns & TDAtt & $\begin{array}{l}\text { All attempts to grapple the opponent to the ground, regardless } \\
\text { of success }\end{array}$ \\
\hline Passes & Passes & $\begin{array}{l}\text { Successfully moving to a more dominant position within grounded } \\
\text { grappling }\end{array}$ \\
\hline Submissions attempted & SubAtt & $\begin{array}{l}\text { All attempts to end the bout through grappling holds, chokes and } \\
\text { joint locks }\end{array}$ \\
\hline
\end{tabular}

$\mathrm{Nb}$. Variable definitions from Kuhn and Crigger ${ }^{27}$ 
The resulting number is the scale of the participant's wingspan in relation to their stature (stature $=1$ ). The difference between bout winners and losers for each variable was calculated by subtracting the bout loser's result from the bout winner's corresponding result to provide the following anthropometric difference variables: AgeDiff, StatDiff, WingspanDiff, S:WDiff; and the following technique difference variables: StrLandedDiff, StrAttDiff, SigStrLandedDiff, SigStrAttDiff, SigDisStrLanDiff, SigDisStrAttDiff, KDownsDiff, DistKDownsDiff, SigClinStrLanDiff, SigGrounStrLanDiff, TDSuccDiff, TDAttDiff, PassesDiff and SubAttDiff.

\section{Statistical analyses}

The following statistical analyses were completed using JASP 0.8.1.1 (JASP Team, Amsterdam, Netherlands) for each competitive division. A Shapiro-Wilk test of normality was calculated for each division $(p \geq 0.05)$. To determine which technique variables the bout winners recorded greater numbers in, the winner and loser technique variable means were compared using a onesided Bayes Factor t-test $\left(\mathrm{BF}_{10}\right)$ using a JZS prior $=$ 0.707 . Due to a default prior being used, a $\mathrm{BF}_{10}$ robustness check was also performed to determine how each result would change under wider priors. The following thresholds were used for categorising each $\mathrm{BF}_{10}: 1-2.9=$ anecdotal; 3-9.9 = moderate; $10-29.9$ = strong; 30=99.9 = very strong; $\geq 100=$ decisive [16]. Where $\mathrm{a} \mathrm{BF}_{10}$ was found to change thresholds in the $\mathrm{BF}_{10}$ robustness check, both threshold categories were reported. To determine to what extent the winner and loser technique variable means were different, Cohen's d effect size (ES) using the standard deviation of the mean scores as the denominator was calculated for each technique variable, using the following thresholds: trivial $\mathrm{d} \leq 0.1$; small $d \geq 0.2$; moderate $d \geq 0.6$; large $d \geq 1.2$, very large $d \geq 2.0$ [28]. Similarly, 95\% credible intervals (95\% CI) were also calculated for each compared variable.
To test the secondary hypothesis of whether anthropometric differences between participants can be used to predict technique use, the anthropometric difference variables were compared to the technique difference variables using either Bayesian Pearson's $r$ or Bayesian Kendall's Tau, depending on whether each variable was parametric or non-parametric. The correlations were set up to test if the variables had a positive correlation only, as the aim of the study was to determine whether, or not, greater anthropometric measurements were related to increased technique success. Any correlations found to have a positive, moderate $\mathrm{BF}_{10}$ or better were then compared using simple linear regression $(p<0.05)$ to determine if a significant prediction equation could be formed.

\section{Ethical approval}

The research related to human use has been complied with all the relevant national regulations and institutional policies, has followed the tenets of the Declaration of Helsinki, and has been approved by the authors' institutional review board or an equivalent committee.

\section{Informed consent}

Informed consent has been obtained from all individuals included in this study.

\section{Results}

To ensure a succinct and manageable presentation of the results, only the $\mathrm{BF}_{10}$ tests which elicited a moderate result or better are reported, however, all test result statistics, $\mathrm{BF}_{10}$ robustness check plots and 95\% CI plots can be viewed in full at https://osf.io/kh8m9/ files/. For the full cohort, the bouts winners' descriptive statistics were: age $=29 \pm 4.1 \mathrm{yrs}$, mass $=73.4 \pm$ $14.5 \mathrm{~kg}$ and stature $=177.8 \pm 8.7 \mathrm{~cm}$. The bouts losers' descriptive statistics were: age $=29.9 \pm 8.7$, mass $=$ $73.7 \pm 14.4 \mathrm{~kg}$ and stature $=177.6 \pm 8.5 \mathrm{~cm}$.

Table 3. $\mathrm{BF}_{10}$, effect sizes and descriptives of moderate or better $\mathrm{HW}$ results

\begin{tabular}{lccccccc}
\hline Variable & $\mathrm{BF}_{10}$ & Likelihood of $\mathrm{H}_{1}$ & Cohen's d & Winners & $95 \% \mathrm{CI}$ & Losers & $95 \%$ CI \\
\hline SigStrLanded & 399 & Decisive & 0.84 & $66.5 \pm 72.8$ & {$[38.3,94.7]$} & $36.5 \pm 30.7$ & {$[24.6,48.4]$} \\
SigStrAtt & 123 & Decisive & 0.75 & $96.8 \pm 92.4$ & {$[60.9,132.6]$} & $63.5 \pm 46.6$ & {$[45.5,81.6]$} \\
SigGrounStrLan & 10 & Strong to Moderate & 0.54 & $10.3 \pm 14.8$ & {$[4.6,16.1]$} & $2.3 \pm 4.1$ & {$[0.7,3.9]$} \\
KDowns & 10 & Strong to Moderate & 0.54 & $0.36 \pm 0.49$ & {$[0.17,0.55]$} & $0.07 \pm 0.26$ & {$[-0.03,0.17]$} \\
SigDistStrLan & 7 & Moderate & 0.5 & $21.2 \pm 24.9$ & {$[11.5,30.8]$} & $13.1 \pm 14.1$ & {$[7.7,18.6]$} \\
DistKDowns & 6 & Moderate & 0.48 & $0.3 \pm 0.5$ & {$[0.1,0.5]$} & $0.1 \pm 0.3$ & {$[-0.03,0.2]$} \\
StrLanded & 5 & Moderate & 0.46 & $66.5 \pm 72.8$ & {$[38.3,94.7]$} & $36.5 \pm 30.7$ & {$[24.6,48.4]$} \\
StrAtt & 4 & Moderate to Anecdotal & 0.45 & $96.7 \pm 92.4$ & {$[60.9,132.6]$} & $63.5 \pm 46.6$ & {$[45.4,81.6]$} \\
\hline
\end{tabular}




\section{HUMAN MOVEMENT}

C. Kirk, Does anthropometry influence technical factors in competitive mixed martial arts?

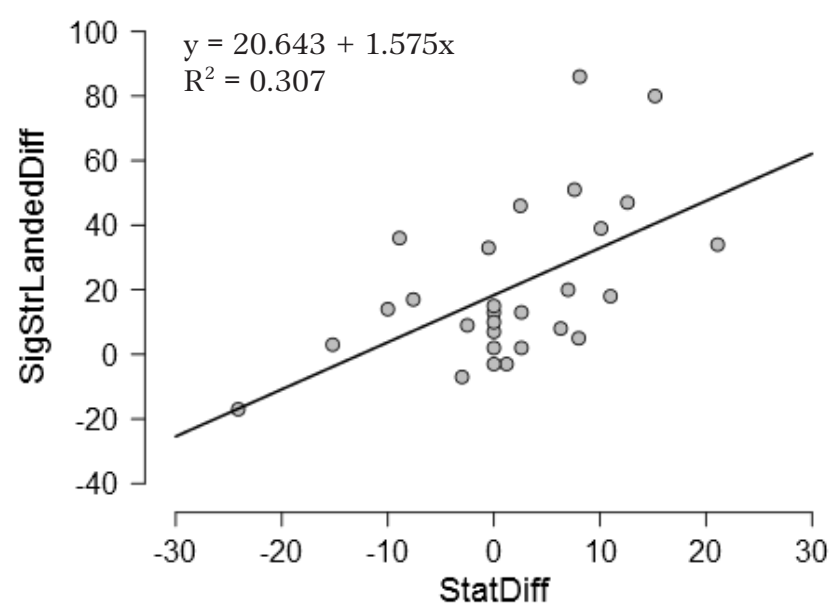

Figure 1a. Bayesian Kendall's Tau between HW StatDiff and SigStrLandedDiff

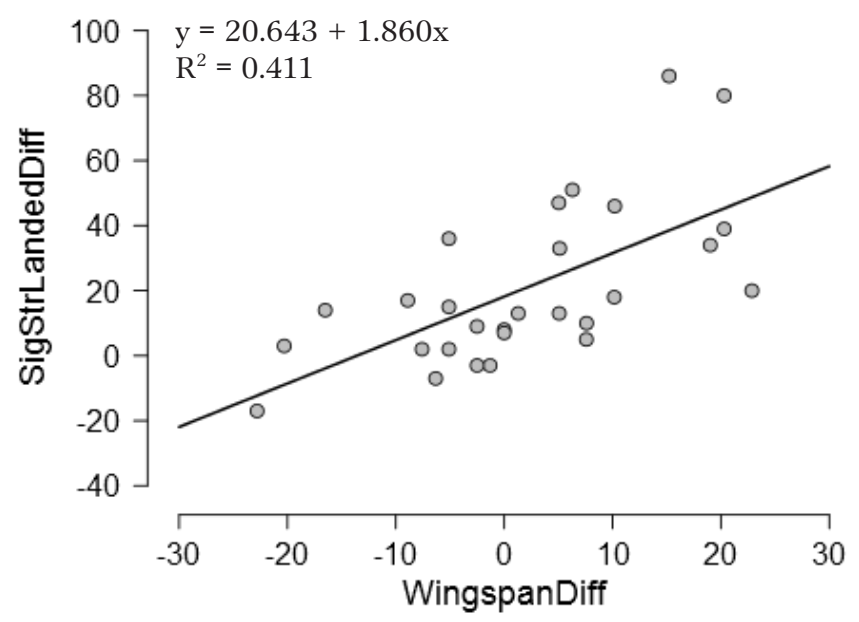

Figure 1b. Bayesian Kendall's Tau between HW WingspanDiff and SigStrLandedDiff

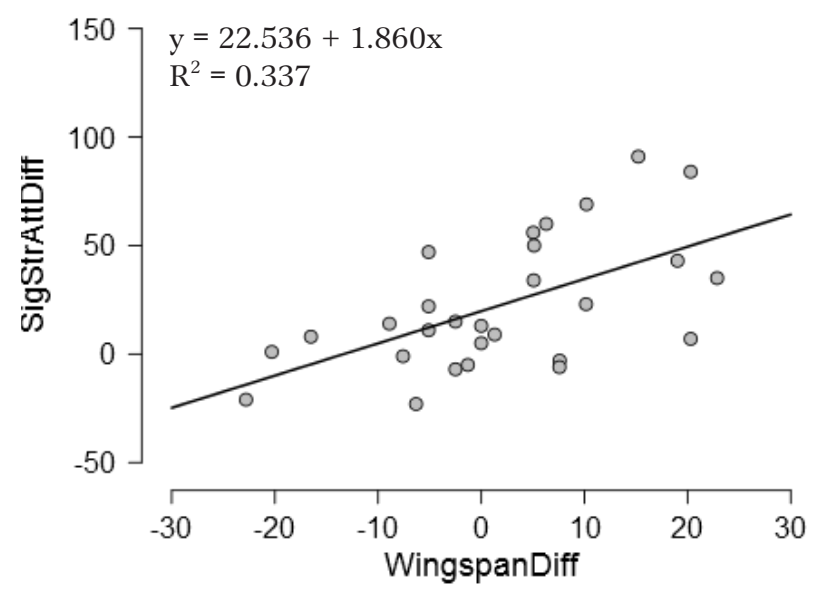

Figure 1c. Bayesian Pearson's r between HW WingspanDiff and SigStrAttDiff
Heavyweight

As presented in Table 3, of the eight variables in which bout winners were more likely to achieve greater numbers, only SigStrLanded and SigStrAtt were decisive according to $\mathrm{BF}_{10}$, and SigStrLanded was the only variable to have a large ES. Three of the variable's $\mathrm{BF}_{10}$ were affected by the robustness check, leaving five variables in the moderate category and one in the anecdotal category. With the exception of SigGrounStrLan, none of the grappling-related variables displayed greater than anecdotal $\mathrm{BF}_{10}$ at $\mathrm{HW}$.

According to Bayesian Kendall's Tau, StatDiff (20.6 \pm $24.6 \mathrm{~cm})$ and SigStrLandedDiff $(1.6 \pm 9.3)$ have a moderate correlation with a strong $\mathrm{BF}_{10}\left(\mathrm{~T}=0.366, \mathrm{BF}_{10}=\right.$ 17.6) (Fig 1a). Linear regression was calculated to predict SigStrLandedDiff based on StatDiff. A significant regression equation was found $\left(\mathrm{F}_{(1,26)}=11.49, \mathrm{p}=0.002\right)$, with $\mathrm{R}^{2}=0.307$, giving the predictive equation of:

Predicted SigStrLandedDiff $=20.643+1.575$ (StatDiff)

WingspanDiff $(1.9 \pm 11.8 \mathrm{~cm})$ was also found to have a moderate correlation with SigStrLandedDiff with a decisive $\mathrm{BF}_{10}$ according to Bayesian Kendall's Tau $\left(\mathrm{T}=0.469, \mathrm{BF}_{10}=170\right)($ Fig $1 \mathrm{~b})$. Linear regression predicts SigStrLandedDiff based on WingspanDiff $\left(\mathrm{F}_{(1,26)}=\right.$ $18.16, p<0.001$ ), with $\mathrm{R}^{2}=0.411$. The predictive equation can be written as:

$$
\begin{gathered}
\text { Predicted SigStrLandedDiff }=20.643+ \\
+1.860 \text { (WingspanDiff })
\end{gathered}
$$

There was also found to be a moderate correlation with a very strong $\mathrm{BF}_{10}$ between WingspanDiff and SigStrAttDiff $(22.5 \pm 30.2)$ according to Bayesian Pearson's $r\left(r=0.581, \mathrm{BF}_{10}=68.5\right)$ (Fig 1c). The subsequent linear regression calculated a significant equation between these two variables $\left(\mathrm{F}_{(1,26)}=13.21, p<0.001\right.$, $\left.\mathrm{R}^{2}=0.337\right)$, given as:

Predicted SigStrAttDiff $=22.536+1.860($ WingspanDiff $)$

\section{Light Heavyweight}

Amongst LHW participants there were seven variables which were calculated to have a moderate or better $\mathrm{BF}_{10}$, all apart from one of which had moderate ES between bout winners and losers (Table 4). Passes displayed a trivial ES but a moderate $\mathrm{BF}_{10}$. StrLanded was the only variable to decisively distinguish between winners and losers. SigGrounStrLan was classed as strong to moderate, while TDSucc and DistKDowns 
were classed as moderate to anecdotal by the $\mathrm{BF}_{10}$ robustness check. No correlations were found between the technique variable differences and the participant anthropometric differences according to Bayesian Pearson's $r$ or Bayesian Kendall's Tau at LHW.

\section{Middleweight}

As shown in Table 5 there was found to be four standing striking related variables classed as decisive and one as very strong. The final decisive variable was SigGrounStrLan. Grappling-related variables also had strong to moderate $\mathrm{BF}_{10}$ at MW. Whilst DistKDowns $\mathrm{BF}_{10}$ was classed as moderate to anecdotal by the $\mathrm{BF}_{10}$ robustness check, there were six variables which had an ES of small or trivial. No correlations between the technique variable differences and the participant anthropometrical differences were found according to Bayesian Pearson's r or Bayesian Kendall's Tau at MW.

\section{Welterweight}

The results at WW (Table 6) contain the greatest amount of decisive results in the study, with eight striking and grappling related variables displaying $\mathrm{BF}_{10}$ greater than 100, and one being classed as decisive to very strong. All ES at WW were moderate or small. Bayesian Kendall's Tau found that StatDiff $(0.4 \pm 6.6 \mathrm{~cm})$ and SigStrAttDiff $(11.1 \pm 30.8) \mathrm{had}$ a moderate, positive correlation with a strong $\mathrm{BF}_{10}$

Table $4 . \mathrm{BF}_{10}$, effect sizes and descriptives of moderate or better LHW results

\begin{tabular}{lccccccc}
\hline Variable & $\mathrm{BF}_{10}$ & Likelihood of $\mathrm{H}_{1}$ & Cohen's d & Winners & $95 \%$ CI & Losers & $95 \%$ CI \\
\hline StrLanded & 105 & Decisive & 0.79 & $69 \pm 50.8$ & {$[48,89.9]$} & $39.8 \pm 36.9$ & {$[24.5,55]$} \\
SigGrounStrLan & 13 & Strong to Moderate & 0.59 & $8.7 \pm 13.9$ & {$[3,14.4]$} & $0.8 \pm 1.7$ & {$[0.07,1.4]$} \\
SigStrLanded & 6 & Moderate & 0.51 & $44.4 \pm 30.2$ & {$[32,56.8]$} & $33.4 \pm 33.4$ & {$[19.6,47.2]$} \\
StrAtt & 5 & Moderate & 0.5 & $117.6 \pm 82$ & {$[83.7,151.4]$} & $88.2 \pm 82.3$ & {$[54.2,122.2]$} \\
Passes & 5 & Moderate & 0.11 & $1.6 \pm 2.7$ & {$[0.4,2.7]$} & $0.3 \pm 0.9$ & {$[-0.05,0.7]$} \\
TDSucc & 4 & Moderate to Anecdotal & 0.47 & $1.6 \pm 2.2$ & {$[0.6,2.5]$} & $0.4 \pm 0.9$ & {$[0.04,0.8]$} \\
DistKDowns & 4 & Moderate to Anecdotal & 0.37 & $0.3 \pm 0.5$ & {$[0.09,0.5]$} & $0.04 \pm 0.2$ & {$[-0.04,0.1]$} \\
\hline
\end{tabular}

Table $5 . \mathrm{BF}_{10}$, effect sizes and descriptives of moderate or better MW results

\begin{tabular}{lccccccc}
\hline Variable & $\mathrm{BF}_{10}$ & Likelihood of $\mathrm{H}_{1}$ & Cohen's d & Winners & $95 \%$ CI & Losers & $95 \%$ CI \\
\hline SigStrLanded & 791661 & Decisive & 0.26 & $39.9 \pm 28.2$ & {$[31.9,47.8]$} & $22.4 \pm 20.3$ & {$[16.7,28.1]$} \\
StrLanded & 21696 & Decisive & 0.76 & $60.9 \pm 44.6$ & {$[48.3,73.4]$} & $32.6 \pm 27$ & {$[25,40.2]$} \\
StrAtt & 750 & Decisive & 0.62 & $102.8 \pm 72.7$ & {$[82.4,123.3]$} & $71.1 \pm 63.4$ & {$[53.3,88.9]$} \\
SigStrAtt & 147 & Decisive & 0.34 & $79.6 \pm 61.7$ & {$[62.1,96.9]$} & $59.6 \pm 59.7$ & {$[42.8,76.4]$} \\
SigGrounStrLan & 471 & Decisive & 0.13 & $12 \pm 18.6$ & {$[6.8,17.3]$} & $1.4 \pm 2.9$ & {$[0.5,2.2]$} \\
SigDisStrLan & 48 & Very Strong & 0.91 & $22.5 \pm 22.6$ & {$[16.1,28.8]$} & $17.3 \pm 17.2$ & {$[12.4,22.1]$} \\
Passes & 25 & Strong & 0.36 & $1.7 \pm 2.1$ & {$[1.1,2.3]$} & $0.6 \pm 1.4$ & {$[0.2,1]$} \\
TDSucc & 6 & Moderate & 0.23 & $1.4 \pm 2.3$ & {$[0.8,2]$} & $0.6 \pm 1.4$ & {$[0.2,1]$} \\
DistKDowns & 4 & Moderate to Anecdotal & 0.45 & $0.2 \pm 0.4$ & {$[0.1,0.4]$} & $0.1 \pm 0.2$ & {$[-0.01,0.1]$} \\
\hline
\end{tabular}

Table $6 . \mathrm{BF}_{10}$, effect sizes and descriptives of moderate or better WW results

\begin{tabular}{|c|c|c|c|c|c|c|c|}
\hline Variable & $\mathrm{BF}_{10}$ & Likelihood of $\mathrm{H}_{1}$ & Cohen's d & Winners & $95 \% \mathrm{CI}$ & Losers & $95 \% \mathrm{CI}$ \\
\hline SigGrounStrLan & $3.533 e+6$ & Decisive & 0.69 & $7.5 \pm 8.6$ & {$[5.7,9.3]$} & $1.3 \pm 2.7$ & {$[0.7,1.8]$} \\
\hline StrLanded & 505088 & Decisive & 0.64 & $59 \pm 42.1$ & {$[50,68]$} & $34.8 \pm 31.3$ & {$[28.2,41.5]$} \\
\hline SigStrLanded & 370517 & Decisive & 0.63 & $35.5 \pm 23.6$ & {$[30.5,40.1]$} & $24.8 \pm 21$ & {$[20.4,29.3]$} \\
\hline Passes & 17100 & Decisive & 0.55 & $1.8 \pm 2.5$ & {$[1.3,2.3]$} & $0.4 \pm 0.9$ & {$[0.2,0.6]$} \\
\hline StrAtt & 7021 & Decisive & 0.53 & $101.6 \pm 65.3$ & {$[87.7,115.6]$} & $75 \pm 59.7$ & {$[62.3,87.7]$} \\
\hline KDowns & 1976 & Decisive & 0.49 & $0.4 \pm 0.5$ & {$[0.3,0.5]$} & $0.1 \pm 0.3$ & {$[0.03,0.2]$} \\
\hline TDSucc & 1996 & Decisive & 0.49 & $1.7 \pm 2.2$ & {$[1.3,2.2]$} & $0.6 \pm 1.1$ & {$[0.4,0.8]$} \\
\hline DistKDowns & 251 & Decisive & 0.42 & $0.3 \pm 0.5$ & {$[0.2,0.5]$} & $0.1 \pm 0.3$ & {$[0.03,0.2]$} \\
\hline TDAtt & 106 & Decisive to Very Strong & 0.39 & $4.1 \pm 4.7$ & {$[3.1,5.5]$} & $1.9 \pm 2.3$ & {$[1.4,2.4]$} \\
\hline SigStrAtt & 41 & Strong & 0.36 & $75.1 \pm 52.7$ & {$[63.9,86.4]$} & $64 \pm 52.2$ & {$[52.9,75.1]$} \\
\hline SigDistStrLan & 21 & Strong & 0.34 & $22.5 \pm 19.1$ & {$[18.4,26.6]$} & $18.9 \pm 18.1$ & {$[15.1,22.8]$} \\
\hline
\end{tabular}




\section{HUMAN MOVEMENT}

C. Kirk, Does anthropometry influence technical factors in competitive mixed martial arts?

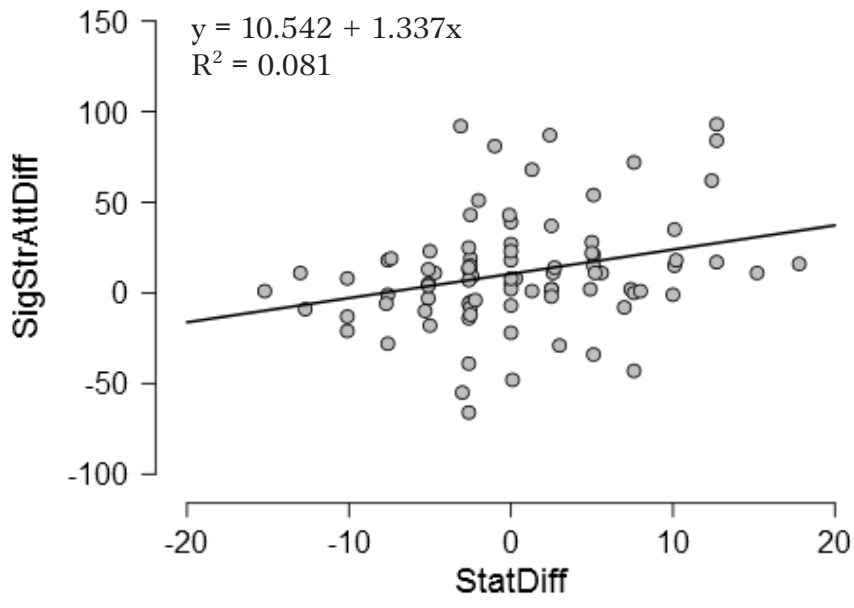

Figure 2a. Bayesian Kendall's Tau between WW StatDiff and SigStrAttDiff

$\left(\mathrm{T}=0.202, \mathrm{BF}_{10}=12\right)$, (Fig 2a). Whilst linear regression calculated a low $\mathrm{R}^{2}=0.081$, the model itself was found to be a significant predictor $\left(\mathrm{F}_{(1,85)}=7.500, p=\right.$ 0.008). The prediction equation can be written as:

Predicted SigStrAttDiff $=10.542+1.337$ (StatDiff)

StatDiff was found to have a moderate correlation with SigDistStrAttDiff $(1.5 \pm 24.1)$ by Bayesian Kendall's Tau with a strong $\mathrm{BF}_{10}\left(\mathrm{~T}=0.209, \mathrm{BF}_{10}=16\right)$ (Fig 2b). Linear regression again calculated a low $\mathrm{R}^{2}=$ 0.068 but a significant prediction model $\left(\mathrm{F}_{(1,85)}=6.212\right.$, $p=0.015)$, giving a prediction equation of:

Predicted SigDistStrAttDiff $=1.049+0.958($ StatDiff $)$

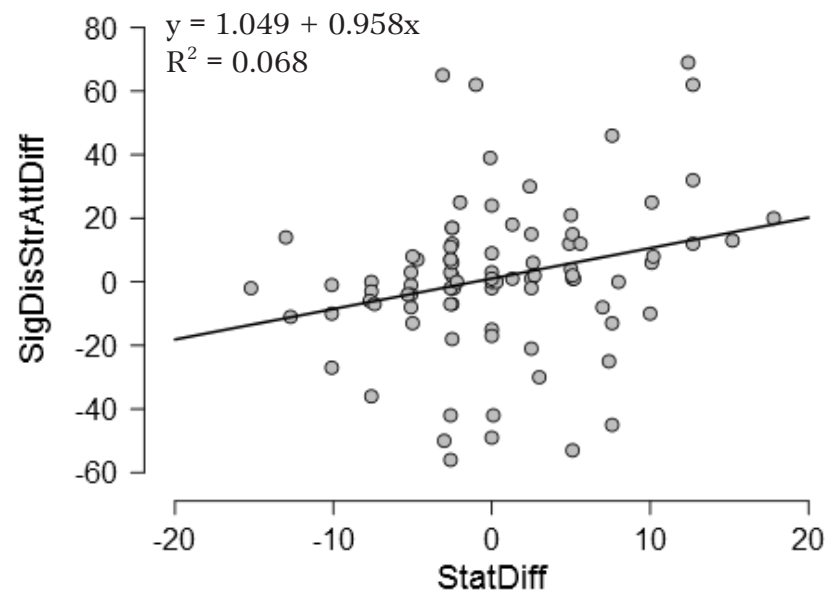

Figure 2b. Bayesian Kendall's Tau between WW StatDiff and SigDistStrAttDiff

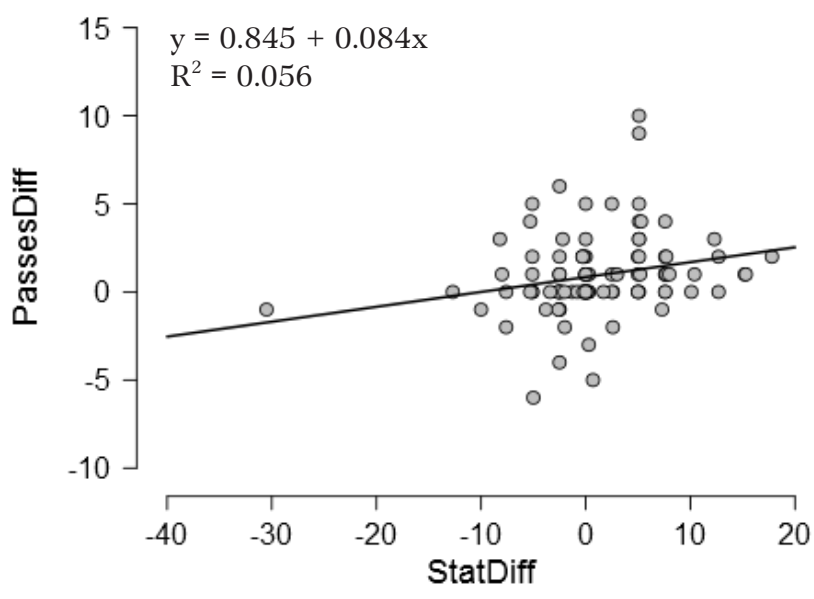

Figure 3a. Bayesian Kendall's Tau between LW StatDiff and PassesDiff

Table 7. $\mathrm{BF}_{10}$, effect sizes and descriptives of moderate or better LW results

\begin{tabular}{lccccccc}
\hline Variable & $\mathrm{BF}_{10}$ & Likelihood of $\mathrm{H}_{1}$ & Cohen's $\mathrm{d}$ & Winners & $95 \% \mathrm{CI}$ & Losers & $95 \% \mathrm{CI}$ \\
\hline SigStrLanded & 322201 & Decisive & 0.86 & $42.4 \pm 30.5$ & {$[36.3,48.5]$} & $29.6 \pm 25.1$ & {$[24.6,34.6]$} \\
SigGrounStrLan & 272457 & Decisive & 0.58 & $6.5 \pm 9.2$ & {$[4.6,8.3]$} & $1.3 \pm 3$ & {$[0.7,2]$} \\
StrLanded & 14770 & Decisive & 0.51 & $60.7 \pm 42.1$ & {$[52.3,69.1]$} & $40.6 \pm 30.4$ & {$[34.6,46.7]$} \\
StrAtt & 14172 & Decisive & 0.51 & $115.5 \pm 74.9$ & {$[100.5,130.4]$} & $87.7 \pm 61.5$ & {$[75.5,100]$} \\
SigStrAtt & 6737 & Decisive & 0.49 & $94.6 \pm 69.7$ & {$[80.7,108.6]$} & $75.3 \pm 58.6$ & {$[63.6,87]$} \\
SubAtt & 6060 & Decisive & 0.49 & $0.5 \pm 0.8$ & {$[0.3,0.6]$} & $0.1 \pm 0.4$ & {$[0.01,0.2]$} \\
KDowns & 5157 & Decisive & 0.48 & $0.3 \pm 0.5$ & {$[0.2,0.4]$} & $0.02 \pm 0.1$ & {$[-0.01,0.05]$} \\
DistKDowns & 1875 & Decisive & 0.45 & $0.3 \pm 0.5$ & {$[0.2,0.3]$} & $0.02 \pm 0.1$ & {$[-0.01,0.05]$} \\
Passes & 309 & Decisive & 0.4 & $1.4 \pm 2$ & {$[1,1.8]$} & $0.5 \pm 1.3$ & {$[0.2,0.7]$} \\
SigDistStrLan & 54 & Very Strong & 0.35 & $30.8 \pm 28.6$ & {$[25.1,36.5]$} & $24.8 \pm 23.8$ & {$[20.1,29.6]$} \\
TDSucc & 36 & Very Strong to Strong & 0.33 & $1.4 \pm 1.7$ & {$[1.1,1.7]$} & $0.7 \pm 1.1$ & {$[0.4,0.9]$} \\
SigClinStrLan & 34 & Very Strong to Strong & 0.33 & $5.1 \pm 6.2$ & {$[3.9,6.4]$} & $3.4 \pm 5$ & {$[2.4,4.4]$} \\
SigDistStrAtt & 10 & Strong to Moderate & 0.3 & $77.6 \pm 69.3$ & {$[63.8,91.4]$} & $67.6 \pm 57$ & {$[56.3,79]$} \\
\hline
\end{tabular}




\section{Lightweight}

Amongst LW participants, bout winners are decisively more likely to attain greater numbers in nine of the measured variables, with a very strong likelihood in three more (Table 7). Whilst four variables recorded moderate ES in favour of bout winners, nine of the reported variables had small ES. TDSucc and SigClinStrLAn were classed as very strong to strong, whilst SigDistStrAtt was classed as strong to moderate by the $\mathrm{BF}_{10}$ robustness check. The only variable to display a correlation to anthropometrical differences at LW was PassesDiff $(0.9 \pm 2.3)$, which had a moderate, positive correlation to Statdiff $(1 \pm 6.5 \mathrm{~cm})$, with a very strong $\mathrm{BF}_{10}$ according to Bayesian Kendall's Tau $\left(\mathrm{T}=0.229, \mathrm{BF}_{10}=68\right)($ Fig 3a) . The resultant linear regression calculated a relatively low $\mathrm{R}^{2}=0.056$ but a significant prediction model $\left(\mathrm{F}_{(1,97)}=5.804, p=0.02\right)$ leading to the following prediction equation:

\section{Predicted PassesDiff $=0.845+0.084($ StatDiff $)$}

\section{Featherweight}

KDowns and DistKDowns were the only decisively distinguishing variables between winners and losers in the FW division (Table 8), both with moderate ES. Whilst SubAtt was classed as very strong, SigStrLanded and SigGrounStrLan were classed as strong to moderate by $\mathrm{BF}_{10}$ robustness check, both with a small ES. When calculating Bayesian Kendall's Tau, StatDiff $(-0.2 \pm 5.3 \mathrm{~cm})$ and KDownsDiff $(0.4 \pm 0.6)$ shared a positive, moderate correlation with a decisive $\mathrm{BF}_{10}(\mathrm{~T}=$ $\left.0.352, \mathrm{BF}_{10}=313\right)(\mathrm{Fig} 4 \mathrm{a})$, and linear regression calculated a significant prediction model $\left(\mathrm{F}_{(1,51)}=7.769\right.$, $p=0.007)$ with $\mathrm{R}^{2}=0.132$. The resultant equation is:

Predicted KDownsDiff $=0.385+0.043$ (StatDiff)

StatDiff was moderately correlated to DistKDownsDiff $(0.3 \pm 0.6)$ with a very strong $\mathrm{BF}_{10}$ according to Bayesian
Kendall's Tau $\left(\mathrm{T}=0.296, \mathrm{BF}_{10}=43\right)(\mathrm{Fig} 4 \mathrm{~b})$. A significant prediction model was found via linear regression $\left(\mathrm{F}_{(1,51)}=5.319, \mathrm{p}=0.02\right)$ with $\mathrm{R}^{2}=0.094$. The prediction equation can be written as:

\section{Predicted DistKDowns $=0.346+0.036($ StatDiff $)$}

WingspanDiff $(0.8 \pm 9.1 \mathrm{~cm})$ also had a positive, moderate correlation to KDownsDiff $\left(\mathrm{T}=0.376, \mathrm{BF}_{10}\right.$ $=805$ ) with a decisive $\mathrm{BF}_{10}$ (Fig $4 \mathrm{c}$ ). With $\mathrm{R}^{2}=0.163$, linear regression calculated a significant prediction model $\left(\mathrm{F}_{(1,51)}=9.919, \mathrm{p}=0.003\right)$ between the two variables, which can be used to predict KdownsDiff using the following equation:

\section{Predicted KdownsDiff $=0.355+0.028$ (WingspanDiff $)$}

A final, decisive correlation was the positive correlation between WingspanDiff and DistKDownsDiff, according to Bayesian Kendall's Tau ( $\mathrm{T}=0.325, \mathrm{BF}_{10}$ $=115)($ Fig $4 \mathrm{~d})$. Linear regression found a significant prediction model to be present $\left(\mathrm{F}_{(1,51)}=7.087, p=0.01\right)$ with $\mathrm{R}^{2}=0.122$. The model's equation can be written as:

Predicted DistKDownsDiff $=0.321+0.024($ WingspanDiff $)$

\section{Bantamweight}

Of the twelve reported variables in the BW division, three resulted in a decisive $\mathrm{BF}_{10}$ in favour of the bout winners, SigStrLanded with a large ES (Table 9). Five of the results were affected by the $\mathrm{BF}_{10}$ robustness check, one being classed as moderate to anecdotal. Non-striking grappling movements were classed as either strong to moderate, moderate or moderate to anecdotal, with the most decisive variables all involving striking. There were no correlations between the technique variable differences and the participant anthropometrical differences according to Bayesian Pearson's r or Bayesian Kendall's Tau at BW.

Table $8 . \mathrm{BF}_{10}$, effect sizes and descriptives of moderate or better $\mathrm{FW}$ results

\begin{tabular}{lccccccc}
\hline Variable & $\mathrm{BF}_{10}$ & Likelihood of $\mathrm{H}_{1}$ & Cohen's d & Winners & 95\% CI & Losers & 95\% CI \\
\hline KDowns & 748 & Decisive & 0.6 & $0.4 \pm 0.6$ & {$[0.3,0.6]$} & $0.03 \pm 0.2$ & {$[-0.01,0.1]$} \\
DistKDowns & 236 & Decisive & 0.55 & $0.4 \pm 0.6$ & {$[0.2,0.5]$} & $0.03 \pm 0.2$ & {$[-0.02,0.1]$} \\
SubAtt & 75 & Very Strong & 0.49 & $0.67 \pm 1.1$ & {$[0.4,1]$} & $0.2 \pm 0.4$ & {$[0.1,0.3]$} \\
SigStrLanded & 38 & Strong to Moderate & 0.46 & $31.2 \pm 22.7$ & {$[24.9,37.5]$} & $23.3 \pm 18.8$ & {$[18.1,28.5]$} \\
SigGrounStrLan & 37 & Strong to Moderate & 0.46 & $6.7 \pm 8$ & {$[4.5,8.9]$} & $2.7 \pm 5.1$ & {$[1.3,4.1]$} \\
Passes & 9 & Moderate & 0.38 & $1.9 \pm 3$ & {$[1.1,2.7]$} & $0.7 \pm 1.3$ & {$[0.3,1]$} \\
StrLanded & 6 & Moderate & 0.26 & $49.2 \pm 36.1$ & {$[39.3,59.1]$} & $36.5 \pm 32$ & {$[27.7,45.4]$} \\
\hline
\end{tabular}




\section{HUMAN MOVEMENT}

C. Kirk, Does anthropometry influence technical factors in competitive mixed martial arts?

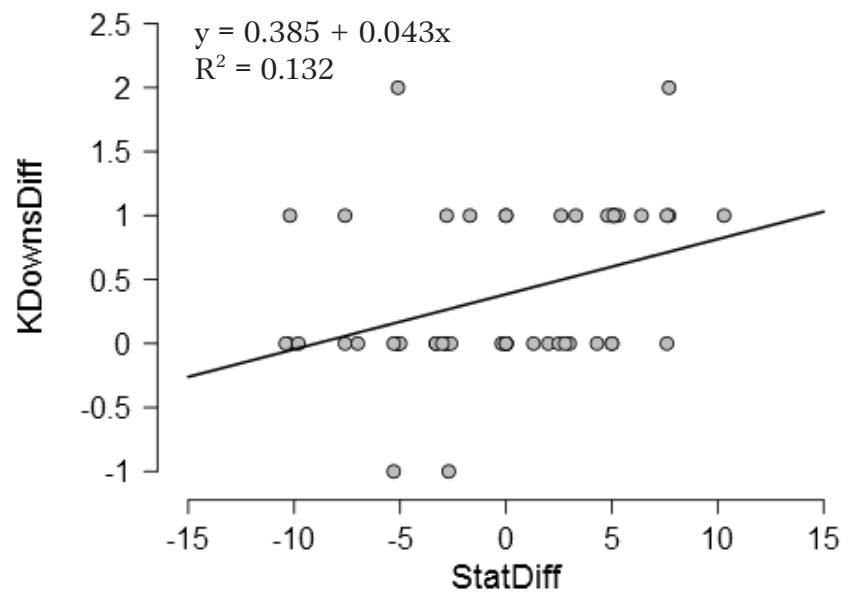

Fig 4a. Bayesian Kendall's Tau between FW StatDiff and KDownsDiff

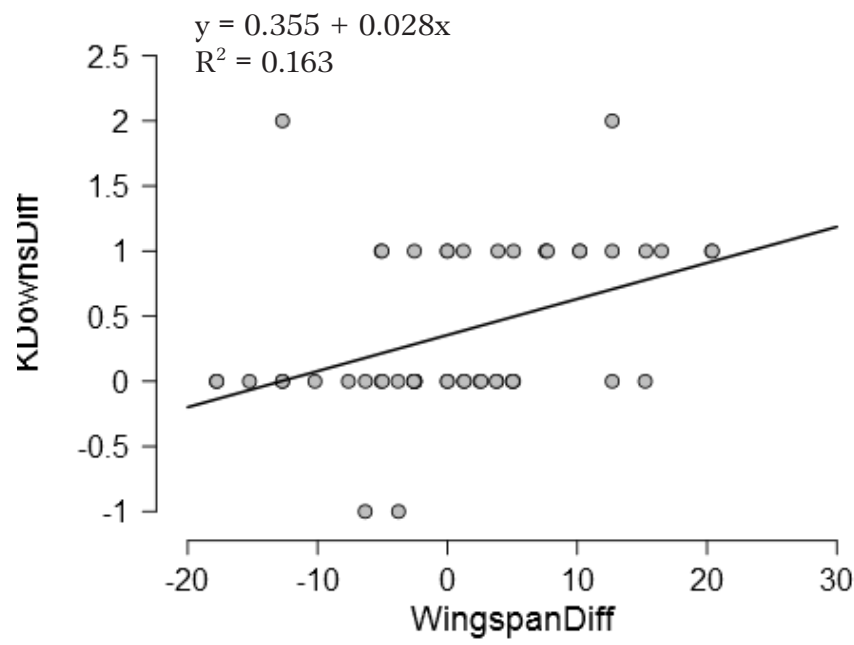

Figure 4c. Bayesian Kendall's Tau between WingspanDiff and KDownsDiff

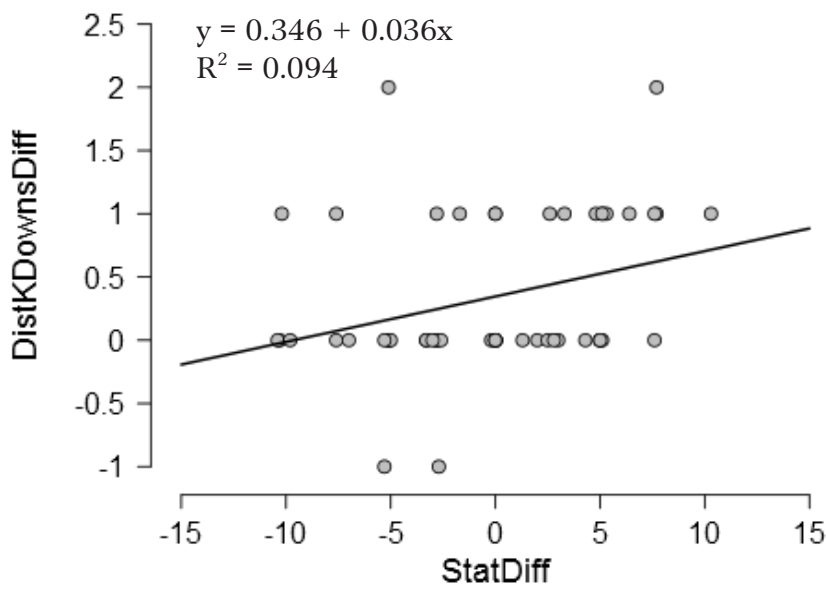

Figure 4b. Bayesian Kendall's Tau between FW StatDiff and DistKDownsDiff

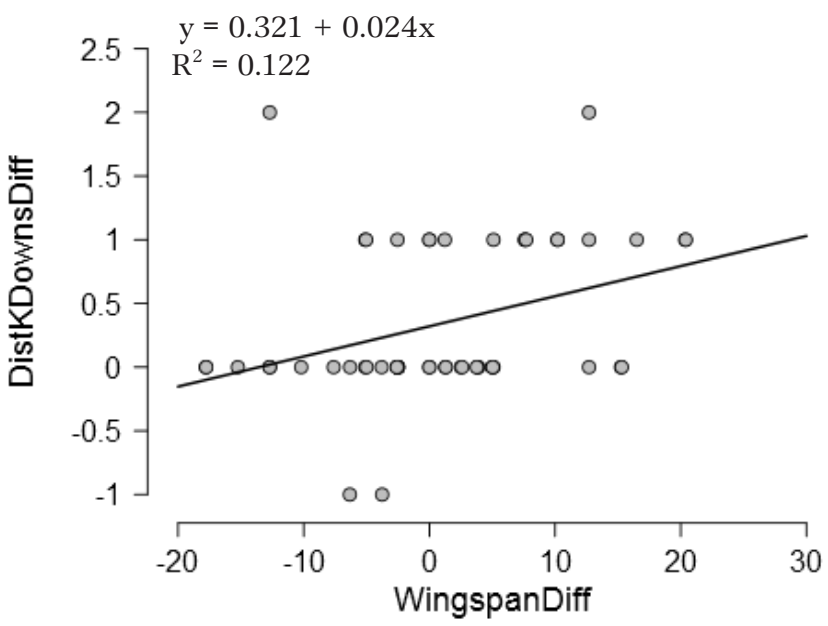

Figure 4d. Bayesian Kendall's Tau between FW WingspanDiff and DistKDownsDiff

Table $9 . \mathrm{BF}_{10}$, effect sizes and descriptives of moderate or better $\mathrm{BW}$ results

\begin{tabular}{lccccccc}
\hline Variable & $\mathrm{BF}_{10}$ & Likelihood of $\mathrm{H}_{1}$ & Cohen's d & Winners & $95 \%$ CI & Losers & $95 \%$ CI \\
\hline SigStrLanded & 178341 & Decisive & 1.08 & $39.6 \pm 26.1$ & {$[30.8,48.4]$} & $26.4 \pm 19.6$ & {$[19.8,33]$} \\
SigGrounStrLan & 217 & Decisive & 0.68 & $5.1 \pm 5.9$ & {$[3.1,7.1]$} & $1.6 \pm 3$ & {$[0.6,2.6]$} \\
SigStrAtt & 159 & Decisive & 0.66 & $88.5 \pm 59$ & {$[68.6,108.5]$} & $71.6 \pm 55.3$ & {$[52.9,90.3]$} \\
SigClinStrLan & 86 & Very Strong & 0.62 & $8.2 \pm 9.8$ & {$[4.9,11.5]$} & $4.2 \pm 5.1$ & {$[2.4,5.9]$} \\
StrAtt & 31 & Very Strong to Strong & 0.55 & $108.5 \pm 68.1$ & {$[85.5,131.6]$} & $86.1 \pm 58$ & {$[66,105.7]$} \\
KDowns & 23 & Strong & 0.53 & $0.5 \pm 0.7$ & {$[0.3,0.7]$} & $0.1 \pm 0.3$ & {$[<0.01,0.2]$} \\
StrLanded & 14 & Strong to Moderate & 0.49 & $57.4 \pm 38.4$ & {$[44.5,70.4]$} & $39.7 \pm 30.1$ & {$[29.5,49.9]$} \\
SigDistStrLan & 12 & Strong to Moderate & 0.48 & $26.3 \pm 22.4$ & {$[18.7,33.9]$} & $20.7 \pm 17$ & {$[14.9,26.4]$} \\
SubAtt & 11 & Strong to Moderate & 0.48 & $0.6 \pm 0.9$ & {$[0.3,0.9]$} & $0.2 \pm 0.7$ & {$[-0.04,0.4]$} \\
DistKDowns & 8 & Moderate & 0.45 & $0.4 \pm 0.6$ & {$[0.2,0.6]$} & $0.1 \pm 0.3$ & {$[-0.01,0.2]$} \\
Passes & 5 & Moderate & 0.41 & $1 \pm 1.8$ & {$[0.4,1.6]$} & $0.4 \pm 1.3$ & {$[0.01,0.9]$} \\
TDSucc & 3 & Moderate to Anecdotal & 0.37 & $1.2 \pm 1.6$ & {$[0.7,1.8]$} & $0.5 \pm 0.9$ & {$[0.2,0.8]$} \\
\hline
\end{tabular}


Flyweight

Decisive to strong $\mathrm{BF}_{10}$ results for five striking and grappling variables were found in the FlW division. The most decisive of which was SigStrAtt (Table 10). Three variables were affected by the $\mathrm{BF}_{10}$ robustness check, but all likelihoods remained moderate or better. The five variables which were classed as moderate also had small ES. Bayesian Pearson's $r$ and Bayesian Kendalls' Tau found no correlations between the technique variable differences and the participant anthropometrical differences in the FIW division.
Women's Bantamweight

WBW contained the least moderate or better $\mathrm{BF}_{10}$ results of all the competitive divisions included in the sample (Table 11). Passes and SigStrLanded were the only variables to moderately distinguish between winners and losers, whilst the other three variables were classed as moderate to anecdotal only. All variables had moderate ES with the exception of SigGrounStrLan which was found to have a small ES. There were no correlations between the technique variable differences and the participant anthropometrical differences according to Bayesian Pearson's $r$ or Bayesian Kendall's Tau in the WBW division.

Table 10. $\mathrm{BF}_{10}$, effect sizes and descriptives of moderate or better FIW results

\begin{tabular}{lccccccc}
\hline Variable & $\mathrm{BF}_{10}$ & Likelihood of $\mathrm{H}_{1}$ & Cohen's d & Winners & $95 \%$ CI & Losers & $95 \%$ CI \\
\hline SigStrAtt & 221 & Decisive & 0.68 & $99.9 \pm 50.9$ & {$[82.7,117.1]$} & $82.3 \pm 47$ & {$[66.4,98.1]$} \\
TDSucc & 103 & Decisive to Very Strong & 0.63 & $2.6 \pm 2.9$ & {$[1.6,3.5]$} & $0.6 \pm 1$ & {$[0.3,1]$} \\
SubAtt & 50 & Very Strong & 0.58 & $1 \pm 1.4$ & {$[0.5,1.4]$} & $0.2 \pm 0.6$ & {$[-0.01,0.4]$} \\
StrAtt & 37 & Very Strong to Strong & 0.56 & $130 \pm 62.4$ & {$[108.9,151.1]$} & $102.2 \pm 52.5$ & {$[84.4,120]$} \\
Passes & 24 & Strong & 0.53 & $3.1 \pm 4$ & {$[1.7,4.4]$} & $0.8 \pm 1.3$ & {$[0.4,1.3]$} \\
SigStrLanded & 15 & Strong to Moderate & 0.5 & $41.1 \pm 21.8$ & {$[33.7,48.5]$} & $30.9 \pm 17.6$ & {$[24.9,36.8]$} \\
SigGrounStrLan & 9 & Moderate & 0.46 & $10 \pm 14.1$ & {$[5.2,14.8]$} & $3.4 \pm 4.4$ & {$[1.9,4.9]$} \\
DistKDowns & 8 & Moderate & 0.45 & $0.3 \pm 0.5$ & {$[0.1,0.5]$} & $0.02 \pm 0.2$ & {$[-0.03,0.1]$} \\
KDowns & 8 & Moderate & 0.45 & $0.3 \pm 0.5$ & {$[0.1,0.5]$} & $0.02 \pm 0.2$ & {$[-0.3,0.1]$} \\
SigDisStrAtt & 8 & Moderate & 0.43 & $76.8 \pm 51.8$ & {$[59.3,94.3]$} & $82.3 \pm 47$ & {$[66.4,98]$} \\
StrLanded & 6 & Moderate & 0.43 & $67.8 \pm 42.6$ & {$[53.4,82.3]$} & $49 \pm 30.3$ & {$[38.7,59.3]$} \\
\hline
\end{tabular}

Table $11 . \mathrm{BF}_{10}$, effect sizes and descriptives of moderate or better WBW results

\begin{tabular}{lccccccc}
\hline Variable & $\mathrm{BF}_{10}$ & Likelihood of $\mathrm{H}_{1}$ & Cohen’s d & Winners & $95 \%$ CI & Losers & $95 \%$ CI \\
\hline Passes & 7 & Moderate & 0.6 & $1.7 \pm 2.3$ & {$[0.6,2.8]$} & $0.6 \pm 1.1$ & {$[0.1,1.1]$} \\
SigStrLanded & 7 & Moderate & 0.59 & $36.2 \pm 19.8$ & {$[26.9,45.5]$} & $24.5 \pm 19.3$ & {$[15.5,33.5]$} \\
StrLanded & 4 & Moderate to Anecdotal & 0.51 & $62 \pm 41.9$ & {$[42.4,81.6]$} & $40.3 \pm 36.2$ & {$[23.3,57.1]$} \\
StrAtt & 4 & Moderate to Anecdotal & 0.51 & $110.8 \pm 68.9$ & {$[78.6,143]$} & $82.6 \pm 55.3$ & {$[56.7,108.5]$} \\
SigGrounStrLan & 3 & Moderate to Anecdotal & 0.49 & $7.3 \pm 10.6$ & {$[2.4,12.2]$} & $1.8 \pm 3$ & {$[0.3,3.2]$} \\
\hline
\end{tabular}

Table $12 . \mathrm{BF}_{10}$, effect sizes and descriptions of moderate or better WSW results

\begin{tabular}{lccccccc}
\hline Variable & $\mathrm{BF}_{10}$ & Likelihood of $\mathrm{H}_{1}$ & Cohen's d & Winners & $95 \% \mathrm{CI}$ & Losers & $95 \%$ CI \\
\hline StrLanded & 349 & Decisive & 0.92 & $105.4 \pm 62.5$ & {$[79,131.8]$} & $54.8 \pm 32.8$ & {$[40.9,68.6]$} \\
StrAtt & 105 & Decisive to Very Strong & 0.81 & $172.2 \pm 95.2$ & {$[132,212.4]$} & $113.9 \pm 80$ & {$[80.1,147.7]$} \\
SigStrLanded & 79 & Very Strong & 0.78 & $61.2 \pm 44.7$ & {$[42.3,80]$} & $34.5 \pm 26.6$ & {$[23.3,45.7]$} \\
Passes & 21 & Strong & 0.65 & $2.5 \pm 3.3$ & {$[1.2,3.9]$} & $0.4 \pm 0.6$ & {$[0.1,0.6]$} \\
SigStrAtt & 16 & Strong & 0.62 & $120 \pm 8$ & {$[83.7,157.8]$} & $90.5 \pm 76.4$ & {$[58.3,122.8]$} \\
SigDistStrLan & 11 & Strong to Moderate & 0.59 & $40.8 \pm 43.6$ & {$[22.4,59.1]$} & $24.7 \pm 23.5$ & {$[14.7,34.6]$} \\
SubAtt & 8 & Moderate & 0.55 & $0.8 \pm 1.2$ & {$[0.3,1.3]$} & $0.2 \pm 0.4$ & {$[0.03,0.4]$} \\
SigGrounStrLan & 7 & Moderate & 0.54 & $8.1 \pm 11.4$ & {$[3.3,12.9]$} & $1.9 \pm 3$ & {$[0.6,3.1]$} \\
TDSucc & 6 & Moderate & 0.52 & $1.6 \pm 1.9$ & {$[0.8,2.4]$} & $0.5 \pm 0.7$ & {$[0.3,0.8]$} \\
\hline
\end{tabular}




\section{Women's Strawweight}

StrLanded and StrAtt were found to be decisive and decisive to very strong (respectively) distinguishing variables between winners and losers at WSW. StrLanded was also calculated to have a large ES (Table 12). Whilst StrAtt and SigDistStrlan were affected by $\mathrm{BF}_{10}$ robustness check, all but four of the nine reported variables were found to have strong or better likelihoods of separating winners and losers at WSW. The reported variables were also a mixture of striking and grappling variables. No correlations between the technique variable differences and the participant anthropometrical differences were found according to Bayesian Pearson's r or Bayesian Kendall's Tau in the WSW division.

\section{Discussion}

The two aims of this study were to determine if the winners of MMA bouts achieve greater numbers in any of the highlighted technique variables in competition, and to calculate whether any of these variables share a relationship with, or are affected by, anthropometrical differences. Whilst it is clear that bout winners generally attain greater numbers in more technique variables than bout losers, in order to come to a conclusion regarding which of these variables have greater impact on the bout outcome, it is important to look at the types of techniques in which bout winners demonstrated the highest likelihood of attaining greater numbers than bout losers. Nine of the ten divisions included at least one variable that decisively favoured the bout winners, with LHW having one and LW having nine, whilst WBW was the only division observed to have no decisive variables. For each of these nine divisions at least one of the ambulatory striking variables was amongst the two most decisive, whilst a grappling-based or ground striking-based variable only appeared in the top two in four divisions. The only occasion when a grapplingbased variable was the most distinguishing factor was Passes in WBW, but this only displayed a moderate likelihood. Pure grappling movements in general are found to only occasionally be decisive factors in distinguishing between winners and losers, with TDSucc, Passes and SubAtt being found to have a decisive $\mathrm{BF}_{10}$ on only four instances in total. Grappling combined with striking seems to be equally as important as grappling on its own, as SigGrounStrLan was also found to be decisive four times as well. Based on these findings, the general picture that has emerged is that striking techniques account for the most important differences between winners and losers.

This finding appears to disagree with the previous research which suggested that grappling was the key determinant of winning and losing in MMA [6, $9,12]$, as the data displayed in this study appears to provide evidence that striking takes precedent. However, it must be noted that the current study uses a univariate method as opposed to a multivariate model, meaning the presented data does not discriminate between isolated actions and combined techniques, for example, the use of strikes to create an opportunity for a takedown attempt. Whilst this limits the use of this data for the complete description of an MMA bout and which combinations of techniques leads to victory, when viewed on a division-by-division basis, certain patterns do emerge. Amongst HW participants it was found that significant strikes landed and attempted were the key factor, whilst KDowns and SigGrounStrLan were secondary. This finding suggests that HW participants use their size and strength to produce powerful strikes which knock their opponents down and then defeat them with strikes on the ground, with grappling movements seeming to have little influence on who wins at HW at all. A similar result was found in the LHW and MW divisions, where strikes account for the key techniques, whilst TDSucc and Passes range from strong to anecdotal. This finding indicates that LHW and MW competitors are using grappling as a means to end the contest after subduing their opponent with strikes in a standing position.

The types and amounts of techniques used seems to change in WW and LW as there are a greater number of techniques which display a decisive $\mathrm{BF}_{10}$ in each of these divisions, including purely grappling based movements. Viewing the results of these two divisions can lead to a visualisation of the types of bouts likely taking place: the bout winner engages in close range striking with their opponent, focussing on a mixture of power strikes and non-power strikes, to either open up opportunities to complete a grappling takedown or achieve a knockdown. From this point they grapple on the ground to move to a better position to finish the bout with strikes or submissions or maintain a dominant position to win each round and a decision overall. This suggests that WW and LW participants are required to have a more complete skill set in order to compete at a high level, whereas from HW to MW, being a competent striker alone can lead to success. This changes again at FW, where the ability to knock an opponent down, either at close range or at a distance, is found to be the key distinguishing factor, with SubAtt providing 
a very strong $\mathrm{BF}_{10}$. Furthermore, with takedowns not featuring at all, it is again suggested that grappling is generally only used to finish the bout in this division. BW competitors also seem to utilise a striking first approach, with SigStrLanded being overwhelmingly the most decisive variable and grappling variables such as SubAtt and TDSucc being only strong to moderate and moderate to anecdotal, respectively.

The seven divisions discussed so far gravitate towards natural groupings according to techniques used. HW, LHW and MW appear to be predominantly striking-based divisions which make use of effective grappling movements sparingly. WW and LW are grouped together as participants in these divisions seem to use striking to allow them to adopt a dominant grappling position to control the bout whilst FW and BW revert to a striking first pattern, though with more differentiation in terms of grappling than the three heaviest divisions. A possible explanation for this delineation could be the differences in stature and body mass present between these groupings [22] and the inherent force and movement velocity differences this causes [29]. The larger participants at the HW to MW levels would produce greater force but lower movement velocity in comparison to the WW to BW participants. They would, therefore, have more success in striking over attempting to close the distance and initiate grappling, as this could increase the likelihood of a larger, slower competitor being dealt a debilitating strike in turn. Whilst strikes by WW - LW participants are potentially less forceful than the larger competitors, the ability to move with greater velocity could allow them to engage in grappling easier and with less risk, making this tactic more efficient. Amongst FW and BW participants, however, the potential further velocity increases could mean that the pattern reverts to being more efficient to strike whilst moving in and out of grappling range for these participants. This cannot, however, explain why FIW seems to share a similar profile to WW and LW, with a mixture of effective striking and grappling being the key determinants of who wins the bout. Further research is required to provide some supporting or contradicting evidence for this conjecture.

Amongst the two female divisions, striking ability again seems to be the determining factor, although WBW only revealed moderate, or moderate to anecdotal, likelihoods, making any reasonable judgements regarding this division difficult. This weakness is very likely due to only 20 bouts being included in the sample from this division, meaning more research will be required for this group of participants. In contrast, at
WSW strikes were decisive in determining the winner of the bout, with grounded grappling in the form of Passes being a strong variable. This finding is reflected in previous research which demonstrated that female competitors who won their bouts via stoppage due to strikes or submission recorded more strikes and more grappling-based movements than those who won via decisions [30]. How the bout comes to be on the ground seems to be less important than it is at WW and LW. As TDSucc only had a moderate effect on the outcome suggests that how effective a participant is it grappling once in a grounded position is more important than it is in a standing position in WSW. A potential explanation for this difference could be the relatively low numbers of females engaging in wrestling and other takedown based sports in comparison to males [31], which translates into fewer female MMA competitors being proficient enough in takedowns to effectively and consistently use them in competition enough to affect the bout outcome.

When taking into the account the ES and 95\% CI for each of the variables in each division, it can also be seen that the bout winners aren't required to achieve a much greater number of each technique to cause a difference between themselves and their opponent, as the majority of variables displayed a moderate ES, with none of the variables displaying a large ES and several being calculated as small. This could be interpreted as evidence that in order to stand a higher likelihood of winning an MMA bout, not only must a participant perform greater numbers of each key technique than their opponent, but it also seems that the quality of these techniques matter, rather than simply the volume performed. However, what constitutes a higher quality and lower quality technique cannot be answered by the current study. It is also clear that which variables are classed as 'key variables' differs from division to division, possibly due to the mass, force and velocity differences of the participants between divisions. Again, it must be made clear, that these groupings and patterns are a result of a univariate approach and can only be used as an indication of what patterns appear, rather than an exact description of how each bout was won or lost, which would require a multivariate approach as employed in other research $[9,31]$.

In terms of the hypothesis that anthropometry influences technique use, it was found that some key technique variables can be predicted from anthropometrical differences. In HW, LW and FW each of these variables displayed a decisive likelihood of favouring the bout winner, whilst the variables at WW had a strong 
likelihood in favour of the bout winner. This suggests that the anthropometrical differences between competitors do play a key role to play in determining the winners of these bouts. This stands in contrast to previous research $[21,22]$ which found that anthropometry does not influence the winners of bouts or a participant's overall ranking, though the referenced studies were conducted using the participant's actual stature, wingspan and S:W. Conversely, the data in this study describes the differences between the competitors in each bout, rather than their raw anthropometrical measurements. This difference in measurements suggests that what matters is how much taller or how greater a wingspan a participant has than their opponent, rather than just being tall, or having a long wingspan in and of itself. Based on this finding, it would be worthwhile revisiting the previously named studies in terms of anthropometrical differences instead of overall measurements. The current study does, however, agree with the previous research that S:W has no effect on performance in MMA and, until contrary evidence is found, need not be of immediate concern for researchers or practitioners. The provided prediction equations may be used by coaches and trainers to predict the influence of a participant's relative anthropometry may have on their performance and plan bout tactics and preparation accordingly. It must be made clear, however, that the correlations in this study are small to moderate and most of the $\mathrm{R}^{2}$ results are also relatively small, so must not be taken as having a decisive or conclusive effect on their own, and should only be applied to the competitive divisions in which they were found. Due to the lack of strong correlations or strong $\mathrm{R}^{2}$ results, whilst these findings provide some evidence of an effect of anthropometry on technique usage and success, much more research is required to fully support the stated hypothesis.

\section{Conclusions}

The variables which are most decisive in distinguishing between winners and losers in MMA bouts differ between divisions, potentially due to force and velocity differences based on divisional mass differences. Whilst preliminary, and based on univariate analyses, the results indicate the existence of the following general groupings: $\mathrm{HW}=$ striking to achieve knockout, little to no grappling influence; LHW - MW = striking to achieve knockout, grappling to attain more dominant position; $\mathrm{WW}-\mathrm{LW}=$ striking to create opportunities for grappling to take opponent down and achieve dominant position for knockout or submission; FW - BW = striking to achieve knockout with grappling to maintain position and distance; FlW = striking to create opportunities for grappling to take opponent down and achieve dominant position for knockout or submission; WBW - WSW = striking to achieve knockout with moderate evidence of grappling to achieve dominant position. Stature and wingspan differences have small to moderate relationships with technique differences in some divisions and can be used to predict some technique differences between opponents, whereas $\mathrm{S}: \mathrm{W}$ differences have no influence on technique differences. Future research on this subject should consider the anthropometrical differences between participants as opposed to raw measurements to elicit more applicable findings.

\section{Limitations}

The anthropometrical data used in this study were those reported by the promoters during MMA events. Therefore, it is not known how or when these measurements were taken. This variable could lead to inaccuracies in the measurements. However, alterations to the recorded measurements would have little impact considering the overall picture that has emerged.

\section{Acknowledgements}

The author wishes to sincerely thank Rami Genauer and the Fightmetric team for providing access to their database, without which, this study would not have been possible.

\section{Disclosure statement}

No author has any financial interest or received any financial benefit from this research.

\section{Conflict of interest}

The authors state no conflict of interest.

\section{References}

1. Gracie R, Danaher J. Mastering Jujitsu. ChamGracie R, Danaher J. Mastering Jujitsu. Champaign: Human Kinetics; 2003.

2. James LP, Haff GG, Kelly VG, Beckman EM. Towards a Determination of the Physiological Characteristics Distinguishing Successful Mixed Martial Arts Athletes: A Systematic Review of Combat Sport Literature. Sports Med. 2016;46(10):1525-1551; doi: 10.1007/ s40279-016-0493-1.

3. NJSAC, [Internet], Mixed Martial Arts Unified Rules of Conduct Additional Mixed Martial Arts Rules 2002, [cited 19/4/17]. Available from: http://www.nj.gov/ lps/sacb/docs/martial.html [date accessed: 19/4/17]. 
4. Lovell DI, Bousson M, McLellan C, The Use of Performance Tests for the Monitoring of Training in Combat Sports: A Case Study of a World Ranked Mixed Martial Arts Fighter. J Athl Enhancement. 2013;2(1); doi:10.4172/2324-9080.1000104.

5. Lenetsky S, Harris N. The Mixed Martial Arts Athlete: A Physiological Profile. Strength Cond J. 2012;34(1): 32-47; doi: 10.1519/SSC.0b013e3182389f00.

6. del Vecchio FB, Hirata SM, Franchini E. A Review of Time-Motion Analysis and Combat Development in Mixed Martial Arts Matches at Regional Level Tournaments. Percept Mot Skills. 2011;112(2):639-648; doi: 10.2466/05.25.PMS.112.2.639-648.

7. Kirk C, Hurst HT, Atkins S. Comparison of the Training Loads of Mixed Martial Arts Techniques in Isolated Training and Open Sparring. J Combat Sports Martial Arts. 2015;6(1):1-6.

8. Miarka B, Vecchio FB, Carney S, Amtmann JA. Comparisons: Technical-tactical and Time-Motion Analysis of Mixed Martial Arts by Outcomes. J Strength Cond Res. 2016;30(7):1975-1984; doi: 10.1519/JSC.00000 00000001287.

9. James LP, Robertson G, Haff GG, Beckman EM, Kelly VG. Identifying the Performance Characteristics of a Winning Outcome in Elite Mixed Martial Arts Competition. J Sci Med Sport. 2017;20(3):296-301; doi: 10.1016/j.jsams.2016.08.001.

10. James LP, Beckman EM, Kelly VG, Haff GG. The Neuromuscular Qualities of Higher and Lower-Level Mixed Martial Arts Competitors. Int J Sports Physiol Perform. 2017;12(5)612-620; doi: 10.1123/ijspp.2016-0373.

11. Miarka B, Coswig VS, Vecchio FBD, Brito CJ, Amtmann J. Comparisons of Time-Motion Analysis of Mixed Martial Arts Rounds by Weight Division. Int J Perform Anal Sport. 2015;15(3):1189-1201; doi:10.1080/2474 8668.2015.11868861.

12. Kirk C, Hurst HT, Atkins S. Measuring the Workload of Mixed Martial Arts Using Accelerometry, Time Motion Analysis and Lactate. Int J Perform Anal Sport. 2015;15(1):359-370; doi: 10.1080/24748668.2015.11 868798 .

13. Miarka B, Brito CJ, Amtmann J. Performance probabilities and outcome effects in technical-tactical factors with bout phase changes of mixed martial arts. Int J Perform Anal Sport. 2017;17(4):510-520; doi: 10.1080/ 24748668.2017.1360103.

14. Cumming G. The New Statistics: Why and How. Psychol Sci.2014;25(1):7-29; doi:10.1177/0956797613504966.

15. van de Schoot R, Kaplan D, Denissen J, Asendorpf JB, Neyer F, van Aken MAG, A gentle introduction to bayesian analysis: applications to developmental research. Child Dev. 2014;85(3):842-860; doi: 10.1111/cdev.12169.

16. Wetzels R, Wagenmakers EJ. A default Bayesian hypothesis test for correlations and partial correlations. Psychon Bull Rev. 2012;19(6):1057-1064; doi: 10.3758/ s13423-012-0295-x.
17. Zabukovec R, Tiidus PM. Physiological and Anthropometric Profile of Elite Kickboxers. J Strength Cond Res. 1995;9(4):240-242.

18. Guidetti L, Musulin A, Baldari C. Physiological Factors in Middleweight Boxing Performance. J Sports Med Phys Fitness. 2002;42(3):309-314.

19. Kankanala V, Gunen EA, Igah AS. Anthropometric Characteristics of Selected Combat Athletic Groups. Br J Sports Med. 2010;44 Suppl 1:i1-i38; doi: 10.1136/bjsm. 2010.078725.126.

20. Chaabene H, Tabben M, Mkaouer B, Franchini E, Negra Y, Hammami M, et al. Amateur boxing: physical and physiological attributes. Sports Med. 2015;45(3):337352; doi: 10.1007/s40279-014-0274-7.

21. Kirk C. The Influence of Age and Anthropometric Variables on Winning and Losing in Professional Mixed Martial Arts. Facta Universitatis: Physical Education and Sport. 2016;14(2):226-236.

22. Kirk C. Does Stature or Wingspan Length Have a Positive Effect on Competitor Rankings or Attainment of World Title Bouts in International and Elite Mixed Martial Arts? Sport Sci Rev. 2016;25(1-2):321-344; doi: 10.1515/ssr-2015-0022.

23. Gabbett TJ. Physiological and Anthropometric Characteristics of Amateur Rugby League Players. Br J Sports Med. 2000;34:303-307; doi: 10.1136/bjsm.34.4.303.

24. Norton K, Olds T. Morphological evolution of athletes over the 20th century: causes and consequences. Sports Med. 2001;31(11):763-783; doi: 10.2165/00007256200131110-00001.

25. Young WB, Newton RU, Doyle TL, Chapman D, Cormack S, Stewart G, et al. Physiological and anthropometric characteristics of starters and non-starters and playing positions in elite Australian Rules Football: a case study. J Sci Med Sport. 2005;8(3):333-345; doi: /10.1016/S1440-2440(05)80044-1.

26. Pieter W. Body Build of Elite Junior Taekwondo Athletes. Acta Kines Uni Tartuensis. 2008;13:99-106.

27. Kuhn R, Crigger K. Fightnomics: The Hidden Numbers and Science in Mixed Martial Arts and Why There's No Such Thing as a Fair Fight. Greybeard 2013

28. Knudson D. Significant and meaningful effects in sports biomechanics research. Sports Biomech. 2009;8(1): 96-104; doi: 10.1080/14763140802629966.

29. Samaras TT. Advantages of Shorter Human Height. In: Samaras TT, editor. Human Body Size and the Laws of Scaling: Physiological, Performance, Growth, Longevity and Ecological Ramifications. New York: Nova Science; 2007. p. 47-61.

30. Miarka B, Coswig V, Brito CJ, Slimani M, Amtmann J, Del Vecchio FB. Comparison of Combat Outcomes: Technical and Tactical Analysis of Female MMA. Int J Perform Analysis Sport. 2017;16(2):539-552.

31. Sisjord MK, Kristiansen E. Elite Women Wrestlers' Muscles: Physical Strength and Social Burden. Int Rev Sociol Sport. 2009;44(2-3):231-246, doi: 10.1177/10126902 09335278. 